\title{
Prevalência de Cardiopatias Congénitas em Portugal \\ em 2015: Dados do Registo Nacional de Anomalias \\ Congénitas
}

\section{Congenital Heart Disease Prevalence in Portugal in 2015: Data from the National Register of Congenital Anomalies}

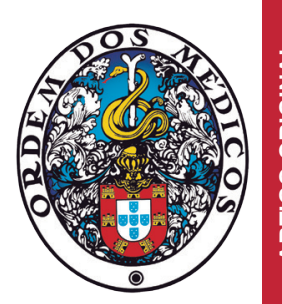

Isabel Saraiva de MELO $\square^{1,2}$, Paula BRAZ³, Rita ROQUETTE ${ }^{3}$, Paulo SOUSA $^{2}$, Carla NUNES 2 , Carlos DIAS ${ }^{2,3}$

Acta Med Port 2020 Jul-Aug;33(7-8):491-499 - https://doi.org/10.20344/amp.12111

RESUMO

Introdução: A prevalência de cardiopatias congénitas em Portugal é de 8,3/1000 nascimentos; cardiopatias congénitas críticas não detectadas podem resultar em graves consequências para o feto/recém-nascido. O objectivo deste trabalho é descrever os casos de cardiopatia congénita reportados em Portugal em 2015 quanto ao diagnóstico pré-natal, patologia cardíaca e à presença de outras malformações congénitas ou anomalias cromossómicas. Estas características são comparadas nos subgrupos dos nados-vivos e de interrupção médica da gravidez. Por último, caracterizam-se os óbitos.

Material e Métodos: Os dados de cardiopatias congénitas reportadas ao Registo Nacional de Anomalias Congénitas em 2015 foram analisados, e calculadas as taxas de prevalência por 1000 nascimentos, comparadas utilizando teste de independência do quiquadrado.

Resultados: A prevalência de cardiopatias congénitas neste estudo foi de 5/1000, (339 nados-vivos, 20\% com cardiopatias congénitas críticas). As cardiopatias mais frequentes foram as seguintes: comunicação interventricular (38\%), comunicação interauricular (15\%), coartação da aorta (7\%), tetralogia de Fallot $(7 \%)$ e estenose pulmonar $(5 \%)$. Um terço dos nados-vivos teve diagnostico pré-natal de cardiopatia. Dos nados-vivos com cardiopatias congénitas críticas, $54 \%$ teve diagnostico pré-natal e $14 \%$ foi diagnosticado ao nascer. Foram identificados 84 registos de interrupção médica da gravidez; $49 \%$ apresentava cardiopatias congénitas críticas, $75 \%$ outras malformações associadas, e $40 \%$ cromossomopatias. Foram registados 15 óbitos (3,4\% de mortalidade) associados a prematuridade e/ou baixo-peso ao nascer, cardiopatias congénitas críticas, outras malformações e anomalias cromossómicas.

Discussão: A prevalência de cardiopatias congénitas neste estudo (5/1000 nascimentos) foi inferior ao descrito noutros estudos internacionais, não obstante uma distribuição por tipo de anomalia semelhante ao previamente reportado. Observaram-se assimetrias regionais significativas que necessitam de mais investigação.

Conclusão: Este estudo é relevante para melhor conhecimento da realidade nacional e organização dos Cuidados de Saúde. É importante uma maior adesão ao Registo Nacional de Anomalias Congénitas.

Palavras-chave: Cardiopatias Congénitas/epidemiologia; Portugal; Registos

\section{ABSTRACT}

Introduction: The prevalence at birth of congenital heart disease in Portugal is 8.3/1000 births; undetected critical congenital heart disease may result in adverse outcomes for the fetus/newborn infant. This study describes the reported cases of congenital heart disease in Portugal in 2015 regarding antenatal diagnosis, cardiac defect, and presence of other congenital anomalies/chromosomal abnormalities. These indicators are compared in live births and medical pregnancy terminations. Additionally, postnatal deaths were characterized.

Material and Methods: Congenital heart disease data derived from the 2015 Portuguese National Registry of Congenital Birth Defects were analyzed. The prevalence rates per 1000 births were assessed by the chi-square test of independence.

Results: The prevalence of congenital heart disease in this study was $5 / 1000$ live-births (339 live-births, $20 \%$ with critical defects). The most common defects were ventricular septal defect (38\%), atrial septal defect (15\%), aortic coarctation ( $7 \%$ ), tetralogy of Fallot $(7 \%)$ and pulmonary stenosis (5\%). One third of the live births had antenatal diagnosis of congenital heart disease. In the live-births with critical congenital heart disease, $54 \%$ had antenatal diagnosis and $14 \%$ were diagnosed at birth. There were records of 84 pregnancy terminations; $49 \%$ had critical defects, $75 \%$ had non-cardiac congenital anomalies and $40 \%$ had chromosomal abnormalities. There were 15 postnatal deaths recorded (3.4\% mortality rate), associated with prematurity/low birthweight, critical congenital heart disease, other non-cardiac congenital anomalies and chromosomal abnormalities.

Discussion: The data analysis revealed a prevalence of congenital heart disease in this study of $5 / 1000$ births (inferior to other international studies), with a distribution per type of anomaly similar to that reported in previously published work. There were significant regional differences that need further studying.

Conclusion: These results are paramount to characterize the Portuguese scenario and improve Healthcare planning. It is important to improve reporting in the Portuguese National Registry of Congenital Birth Defects.

Keywords: Heart Defects, Congenital / epidemiology; Portugal; Registries

\section{INTRODUÇÃO}

As cardiopatias congénitas são as malformações congénitas mais frequentes em Portugal e no mundo. ${ }^{1} \mathrm{~A}$ pre-

valência habitualmente citada é de oito por 1000 nados-vivos. ${ }^{1-3}$ Os dados nacionais mais recentes são do Registo

1. Pediatria. Clínica CUF Almada. Almada. Portugal.

2. Centro de Investigação em Saúde Pública. Escola Nacional de Saúde Pública. Universidade NOVA de Lisboa. Lisboa. Portugal.

3. Departamento de Epidemiologia. Instituto Nacional de Saúde Doutor Ricardo Jorge. Lisboa. Portugal.

$\square$ Autor correspondente: Isabel Saraiva de Melo. ic.melo@ensp.unl.pt

Recebido: 22 de junho de 2019 - Aceite: 13 de novembro de 2019 | Copyright @ Ordem dos Médicos 2020 
Nacional de Anomalias Congénitas (RENAC) de 2014 2015, que apontam para uma prevalência de 8,3 por 1000 nascimentos de malformações cardíacas nesse período. ${ }^{3}$

Estima-se que uma em cada quatro cardiopatias congénitas seja crítica, definida como um defeito cardíaco em que é necessária alguma intervenção (cirúrgica ou percutânea) no primeiro ano de vida. ${ }^{4}$ Uma definição mais estrita de cardiopatias congénitas críticas (CCC) restringe-se aos defeitos congénitos cardíacos ducto-dependentes, potencialmente fatais, em que ocorre morte ou é necessária intervenção cirúrgica ou percutânea no primeiro mês de vida. ${ }^{5,6}$

Apesar dos avanços no diagnóstico prénatal (DPN), nem todas as cardiopatias congénitas críticas (CCC) são passíveis de diagnóstico in utero; por outro lado, nem todas as mulheres têm uma adequada vigilância das suas gravidezes. ${ }^{7,8}$ Adicionalmente, tendo em conta as profundas alterações na fisiologia cardiovascular na adaptação à vida extrauterina, algumas formas de cardiopatia apenas se tornam sintomáticas no final da primeira semana de vida, após o encerramento espontâneo do canal arterial. ${ }^{4,6}$ Inclusive, o choque cardiogénico ou a morte súbita no período neonatal podem constituir a apresentação inicial da CCC. ${ }^{8,9}$

$\mathrm{O}$ rastreio de cardiopatias congénitas por oximetria de pulso antes da alta da maternidade tem como objectivo identificar precocemente os casos de CCC sem DPN e não detectados no exame físico pós-natal, antes que ocorra a descompensação cardíaca. ${ }^{6,10}$ Os recém-nascidos detectados por este rastreio poderão ser imediatamente orientados para o tratamento necessário (cirurgia e/ou intervenção percutânea) em condições clínicas mais favoráveis, com melhores resultados imediatos e a longo prazo., ${ }^{4,11}$

A melhoria na sobrevida dos recém-nascidos e lactentes portadores de CCC tem implicações relevantes na prestação e na organização dos cuidados de saúde. ${ }^{12,13}$ Estas crianças, jovens e adultos devem manter seguimento cardiológico (muitas vezes multidisciplinar) toda a sua vida, necessitando por vezes de múltiplas reintervenções. ${ }^{13}$

O aprofundamento do conhecimento epidemiológico da realidade nacional permitirá adequar e organizar os recursos disponíveis com vista a maior abrangência do diagnóstico prénatal e/ou, pós-natal, e de uma adequada prestação de cuidados aos recém-nascidos, lactentes, crianças e adultos portadores de cardiopatia congénita.

Este estudo teve como principal objectivo o de estimar a prevalência de cardiopatias congénitas, em 2015 em Portugal. Um objectivo secundário foi de estimar também, no mesmo período de tempo, a prevalência de CCC. Neste contexto foi caracterizado o momento do diagnóstico (DPN, ao nascer ou mais tardio), o desenlace da gravidez (nado-vivo, aborto espontâneo, feto-morto ou interrupção da gravidez), o tipo de cardiopatia, o diagnóstico cardíaco, a presença de outras malformações associadas e a presença de anomalias cromossómicas. Foram analisados os casos de CCC quanto à distribuição regional, principais diagnósticos, DPN, presença de outras malformações congénitas e anomalias cromossómicas. Foram comparados os casos de interrupção médica da gravidez com os nados-vivos portadores de cardiopatia congénita quanto ao sexo do feto, presença de cardiopatia crítica, tipo de cardiopatia, presença de outras malformações e de anomalias cromossómicas. Finalmente, foram caracterizados os óbitos registados quanto a idade no óbito, DPN, idade gestacional, peso ao nascer, diagnóstico cardíaco, alterações genéticas e malformações não-cardíacas associadas.

\section{MATERIAL E MÉTODOS}

Os autores realizaram um estudo transversal utilizando o total de casos de cardiopatia congénita reportados ao RENAC em 2015.

\section{Registo Nacional de Anomalias Congénitas}

O Registo Nacional de Anomalias Congénitas (RENAC) é um registo nosológico de base populacional que recebe notificações da ocorrência de anomalias congénitas no Continente e Regiões Autónomas dos Açores e da Madeira desde 1997. Este tem um carácter voluntário, consistindo no preenchimento pelos profissionais de Saúde, e junto dos progenitores, de um questionário com informação relativa à gravidez, consumos maternos, antecedentes familiares, diagnóstico pré-natal, parto, diagnósticos e resultados da autópsia. Atualmente, todas as maternidades nacionais têm elementos de ligação com o RENAC (dos serviços de Obstetrícia e Pediatria/Neonatalogia). A metodologia utilizada pelo RENAC pode ser consultada nos vários relatórios publicados. ${ }^{3,14}$ Os diagnósticos são codificados pelos médicos referenciadores utilizando a $10{ }^{a}$ versão da Classificação Internacional de Doenças (ICD-10), com base na informação clínica, diagnóstico pré-natal, exames complementares pós-natais e dados da autópsia. Em cerca de $10 \%$ dos casos a codificação das anomalias é feita pelos investigadores do Instituto Nacional de Saúde Dr. Ricardo Jorge (INSA), em articulação com os notificadores locais.

Os dados recolhidos são anónimos, e só o elemento de ligação em cada instituição poderá associar o processo clínico do doente à informação na base de dados do RENAC. Foi efectuado um pedido formal de acesso aos dados, que foi devidamente autorizado em novembro de 2017.

Para este estudo foi feita uma extração da base de dados de 2015 tendo como critério de inclusão o diagnóstico de cardiopatia congénita (códigos ICD-10 Q20 a Q26). Foram solicitadas as variáveis data e local de nascimento, sexo, nascimento, desenlace da gestação, peso ao nascer, idade gestacional (em semanas), quando foi identificada a primeira anomalia (pré-natal, ao nascer, primeira semana de vida, entre uma e quatro semanas de vida, autópsia ou desconhecido), estado do feto/RN, primeiro exame pré-natal alterado, resultados morfológicos, sobrevivência além da primeira semana, data da morte, autópsia, descrição das anomalias presentes. Foram ainda incluídos os diagnósticos de outras malformações (não cardíacas), e a presença de anomalias cromossómicas associadas.

Este estudo corresponde a uma análise de dados secundária do conjunto de dados do Registo Nacional de 
Anomalias Congénitas. O RENAC fornece dados anónimos para estudos epidemiológicos, sendo solicitado por escrito pelo investigador quais os dados que deseja analisar. O anonimato implica a remoção de quaisquer dados pessoais. De acordo com a Comissão de Ética para Investigação Clínica, "os dados pessoais que tenham sido tornados anónimos de modo a que a pessoa não seja ou deixe de ser identificável deixam de ser considerados dados pessoais, e por isso não são abrangidos pelo Regulamento Geral de Proteção de Dados". ${ }^{15}$

\section{Recodificação dos casos}

A partir do hospital do registo, os casos foram codificados, em oito regiões, i.e. Administrações Regionais de Saúde (ARS): Norte, Centro, Lisboa e Vale do Tejo (LVT), Alentejo, Algarve, e as Regiões Autónomas dos Açores e da Madeira. Foi considerada vigilância ecográfica da gravidez adequada de acordo com os critérios da Direção Geral de Saúde ${ }^{7}$ : ecografia do primeiro trimestre realizada entre 11 e as 13 semanas de gestação e ecografia do segundo trimestre entre as 20 e 22 semanas, caso a gestação tenha ultrapassado esta duração.

De acordo com os diagnósticos apresentados os casos foram codificados em CCC ou não críticas. Foi definida CCC como os alvos primários e secundários do rastreio de cardiopatias por oximetria de pulso nas seguintes patologias: atrésia da artéria pulmonar, atrésia da válvula tricúspide, retorno venoso pulmonar anómalo total, síndrome do coração esquerdo hipoplásico, tetralogia de Fallot, d-transposição das grandes artérias, truncus arteriosus, anomalia de Ebstein, coartação da aorta, interrupção do arco aórtico, ventrículo direito de dupla saída e ventrículo único (Tabela 1). ${ }^{9}$

Os defeitos cardíacos foram ainda classificados como: shunt esquerdo-direito, obstáculo esquerdo, obstáculo direito, fisiologia de transposição, defeitos complexos e outros (Tabela 2).

\section{Análise estatística}

As taxas de prevalência foram calculadas utilizando no numerador os nados-vivos, interrupções médicas da gravidez (IMG) e fetos mortos com cardiopatia reportados ao RENAC em 2015 pela Administração Regional de Saúde; no denominador nacional o número total de nascimentos (nados-vivos e fetos mortos); nos denominadores regionais a soma dos nados-vivos e óbitos fetais por residência da mãe nas diferentes regiões de saúde de acordo com os dados de 2015 publicados pelo Instituto Nacional de Estatística. ${ }^{16,17}$

Como primeira abordagem, para caracterização dos resultados foram utilizadas tabelas de frequências e tabelas de contingência em que foi aplicado o teste de independência do qui-quadrado $\left(\chi^{2}\right)$. Para comparação entre grupos, nados-vivos e IMG, considerando as características (ARS do hospital de registo, sexo, vigilância obstétrica e DPN, presença de CCC, outras malformações associadas e anomalias cromossómicas), foram efectuadas tabelas de frequências cruzadas e aplicado o teste de independência do qui-quadrado. Para a comparação entre diferentes regiões foram utilizados testes de comparações de proporções duas a duas regiões, assumindo um nível de significância estatística de 5\%. Foi ainda feita a comparação da frequência dos diferentes diagnósticos e tipos de cardiopatia nos nados-vivos e IMG. Foram comparados os grupos com cardiopatia congénita crítica ou não crítica quanto ao registo de DPN na gravidez.

Foi utilizado o software IBM SPSS v. 24 para a análise estatística, e considerado um nível de significância de 0,05.

\section{RESULTADOS \\ Resultados gerais}

Em 2015 foram notificados no RENAC 435 casos portadores de cardiopatia congénita, para um número total de nascimentos (nados-vivos, fetos-mortos e IMG) de $85797 .^{3}$ A maioria dos casos correspondem a nados-vivos $(78 \%)$, e $19 \%$ a IMG; estão ainda registados seis casos de aborto

Tabela 1 - Cardiopatias congénitas classificadas como críticas $^{9}$; ICD-10 - $10^{\text {a }}$ classificação internacional de doenças

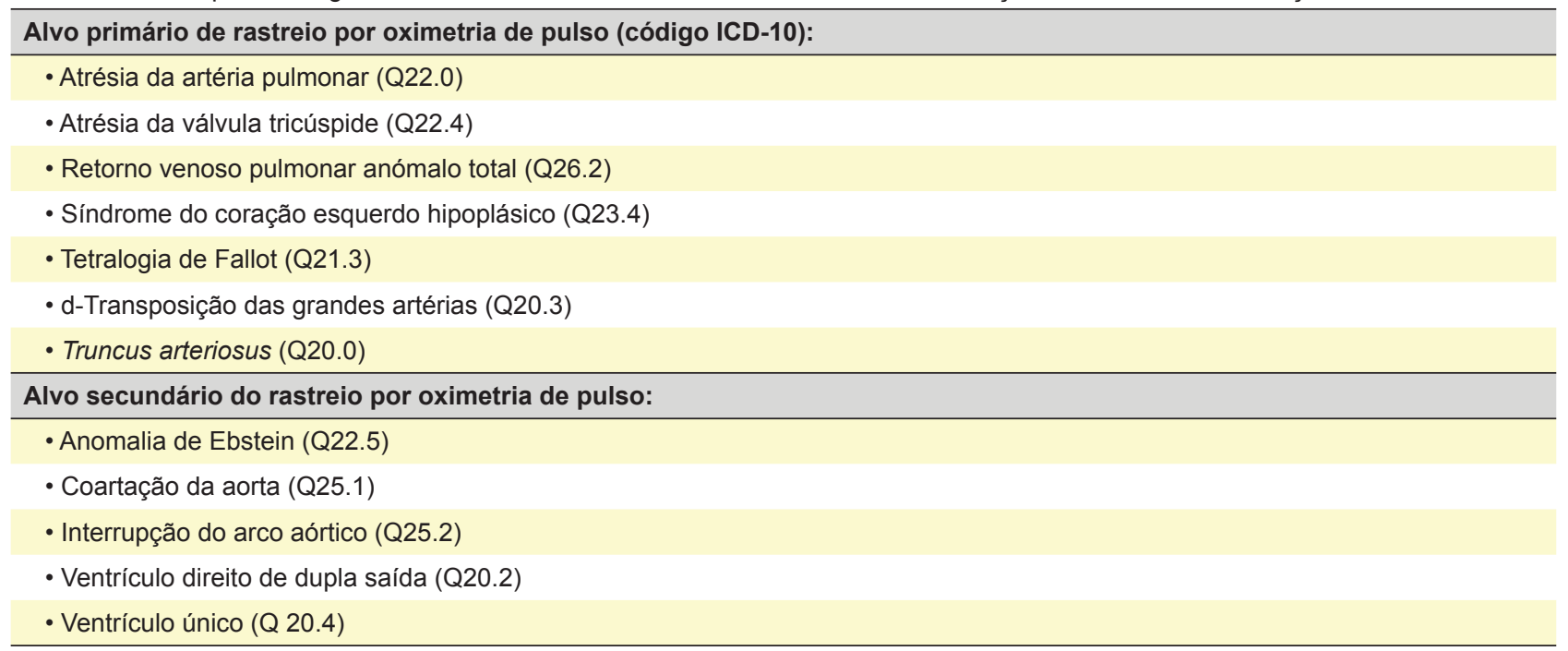


Tabela 2 - Classificação fisiopatológica dos casos de cardiopatia congénita identificados. ICD-10 - $10^{a}$ classificação internacional de doenças

\begin{tabular}{|c|c|}
\hline Tipo & Diagnósticos (códigos ICD-10) \\
\hline Shunt esquerdo-direito & $\begin{array}{l}\text { 1. Comunicação interauricular - CIA / Foramen ovale patente FOP (Q21.1) } \\
\text { 2. Comunicação interventricular - CIV (Q21.0) } \\
\text { 3. Persistência do canal arterial - PCA (Q25.0) } \\
\text { 4. Defeito do septo auriculoventricular DSAV (Q21.2) } \\
\text { 5. Ventrículo único (Q20.4) } \\
\text { 6. Truncus arteriosus (Q20.0) }\end{array}$ \\
\hline Obstáculo esquerdo & $\begin{array}{l}\text { 1. Estenose aórtica (Q23.0) } \\
\text { 2. Coartação da aorta (Q25.1) } \\
\text { 3. Estenose mitral (Q.23.2) } \\
\text { 4. Interrupção do arco aórtico (Q25.2) }\end{array}$ \\
\hline Obstáculo direito & $\begin{array}{l}\text { 1. Estenose pulmonar (Q22.1) } \\
\text { 2. Estenose tricúspide (Q22.4) } \\
\text { 3. Anomalia de Ebstein (Q22.5) }\end{array}$ \\
\hline Fisiologia de transposição & 1. d-Transposição dos grandes vasos (Q 20.3) \\
\hline Complexas & $\begin{array}{l}\text { 1. Tetralogia de Fallot (Q21.3) } \\
\text { 2. Atrésia da artéria pulmonar (Q22.0) } \\
\text { 3. Síndrome do coração esquerdo hipoplásico - SCEH (Q23.4) } \\
\text { 4. Retorno venoso pulmonar anómalo total - RVPAT (Q26.2) } \\
\text { 5. Ventrículo direito de dupla saída (Q20.1) }\end{array}$ \\
\hline Outras & $\begin{array}{l}\text { 1. Veia cava superior esquerda (Q26.1) } \\
\text { 2. Arco aórtico direito (Q25.41) } \\
\text { 3. Dextrocárdia (Q24.0) } \\
\text { 4. I-Transposição dos grandes vasos (Q20.5) } \\
\text { 5. Outras }\end{array}$ \\
\hline
\end{tabular}

espontâneo (antes das 20 semanas) e seis casos de feto-morto (1,4\%). A prevalência de cardiopatias congénitas em Portugal em 2015 calculada neste estudo foi de 5,1 casos por 1000 nascimentos (Tabela 3).

Verificou-se neste estudo uma assimetria regional considerável na prevalência de cardiopatias congénitas: 3/1000 no Alentejo e Lisboa e Vale do Tejo, 4:1000 no Norte e Açores, 5:1000 na Madeira, 6:1000 na região Centro e 7:1000 no Algarve, diferenças com significado estatístico (Tabela 3). Observaram-se ainda diferenças regionais estatisticamente significativas $(p<0,001)$ no registo da detecção pré-natal das cardiopatias (incluindo todos os casos), comparando as regiões Norte, LVT e Algarve (com frequência média de DPN de $55 \%$, superior à media nacional de $47 \%$ ) com as regiões Centro, Algarve, Açores e Madeira (frequência média de DPN de 29\%). A IMG foi também assimétrica nas diferentes regiões: dos casos registados com DPN, houve $0 \%$ de interrupções nos Açores e Madeira, $26 \%$ em Lisboa e Vale do Tejo, $45 \%$ no Centro, $47 \%$ no Algarve e $49 \%$ de interrupções no Norte; as únicas diferenças com significado estatístico foi entre a região norte e a média nacional, regiões Centro, LVT e Alentejo.

Não se observou neste estudo predomínio de nenhum dos sexos; registou-se um caso de ambiguidade sexual, associado a mal-posição das grandes artérias, com comunicação interventricular (CIV), e outras malformações associadas (agenésia do corpo caloso, micrognatismo e talipes equinovarus).

A maioria das grávidas teve acompanhamento pré-natal e vigilância ecográfica da gravidez adequada (85\%); em $10 \%$ não foi registada a realização de qualquer ecografia obstétrica. Em quase metade dos casos totais ( $n=203$,
$46 \%$ ) o diagnóstico foi efectuado no período pré-natal, embora nos nados-vivos esse número diminua para $33 \%$. 0 ecocardiograma fetal está registado em apenas 47 casos; foi considerado normal, ou seja falso-negativo, em $30 \%$ dos casos (14), dois dos quais com defeito crítico (coartação da aorta).

Os diagnósticos mais frequentes de cardiopatia congénita estão ilustrados na Tabela 4.

$\mathrm{Na}$ maioria dos casos (46\%) o diagnóstico foi feito no período pré-natal, em 29\% (126 casos) na primeira semana de vida, 16\% (71 casos) ao nascer, 6\% (25 casos) entre a primeira e quarta semana de vida e $1 \%$ (quatro casos) na autópsia; em $1 \%$ (seis casos) o momento do diagnóstico é desconhecido. Dos quatro casos diagnosticados na autópsia, três corresponderam a feto-morto no segundo trimestre (idades gestacionais entre 15 e 16 semanas) e o quarto a um feto-morto de 36 semanas de idade gestacional, com coartação da aorta.

\section{Cardiopatias críticas}

Dos casos notificados, vinte e cinco por cento (109) corresponderam a CCC; como tal, a prevalência neste estudo de CCC em Portugal em 2015 foi de 1,3:1000 nascimentos. A maioria dos casos foram registados na região Norte (54 casos ou 47\%), seguidos de Lisboa e Vale do Tejo (32 casos ou $28 \%$ ), e Centro (16 casos ou 14\%); não foram registadas cardiopatias críticas no Alentejo; estas diferenças regionais tiveram significado estatístico (valor de $p=$ 0,002), não se observando predomínio de um dos sexos. A presença de outras malformações em $36 \%$ dos casos e de anomalias cromossómicas em $11 \%$ não apresentou diferença estatisticamente significativa comparativamente ao 
Tabela 3 - Resultados globais

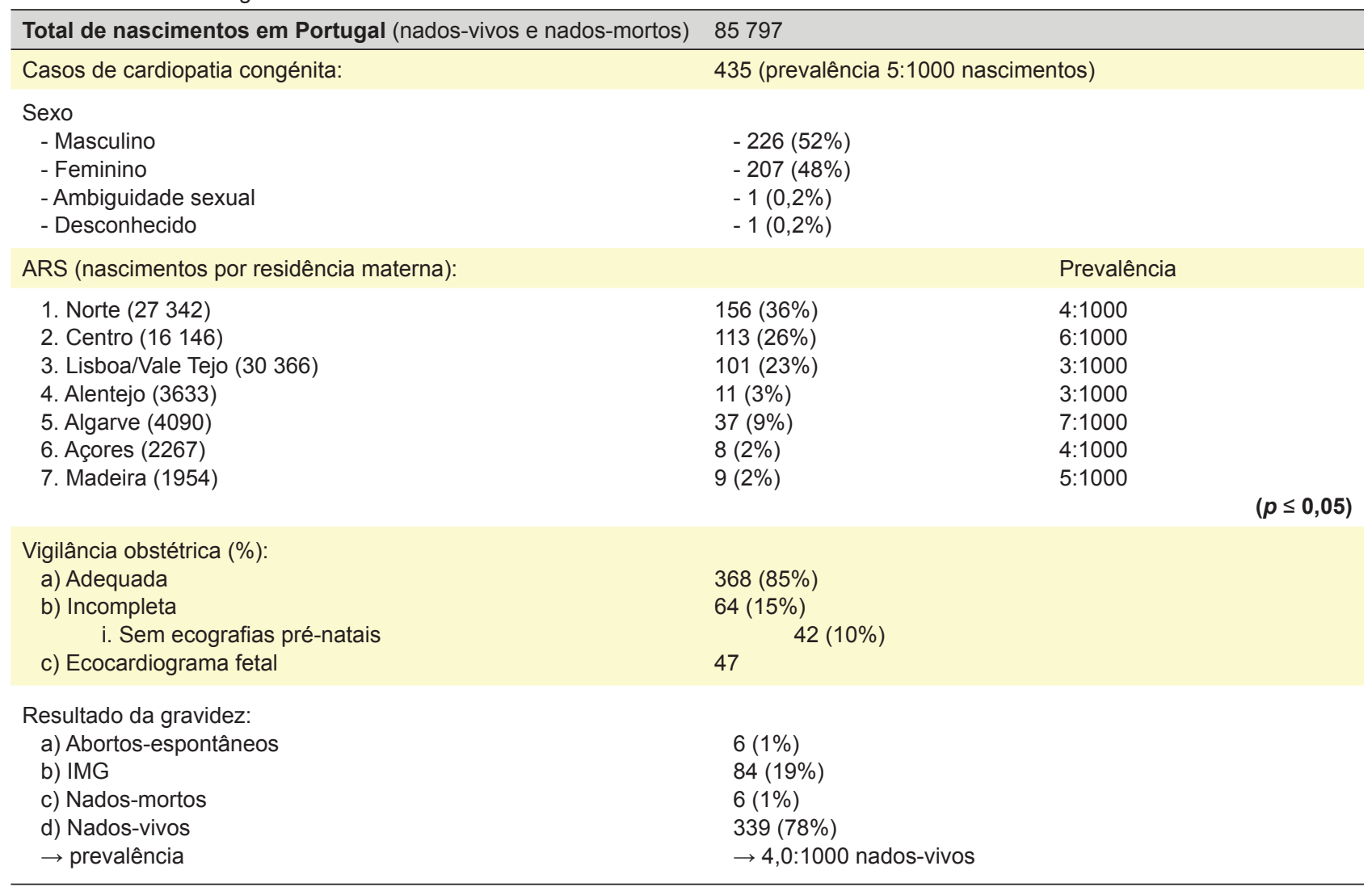

IMG: interrupção médica da gravidez

Tabela 4 - Cardiopatias congénitas críticas: frequências e respectivo diagnóstico prénatal (DPN)

\begin{tabular}{lcc}
\hline Diagnóstico & n (\%) & DPN \\
\hline Coartação da aorta & $32(28 \%)$ & $19 / 32$ \\
Tetralogia de Fallot & $28(25 \%)$ & $19 / 28$ \\
Atrésia da artéria pulmonar & $16(14 \%)$ & $11 / 11$ \\
Síndrome do coração esquerdo hipoplásico & $12(10 \%)$ & $12 / 12$ \\
Transposição grandes vasos & $11(10 \%)$ & $8 / 11$ \\
Truncus arteriosus & $5(4 \%)$ & $5 / 5$ \\
Ventrículo direito de dupla saída & $5(4 \%)$ & $5 / 5$ \\
Retorno venoso pulmonar anómalo total & $4(3,5 \%)$ & $2 / 4$ \\
Anomalia de Ebstein & $1(1 \%)$ & $0 / 1$ \\
TOTAL & 114 & $82(72 \%)$ \\
\hline
\end{tabular}

observado nas cardiopatias não críticas. A cardiopatia crítica mais frequentemente registada foi a coartação da aorta (32 casos), seguida da tetralogia de Fallot (28 casos) e da atrésia da artéria pulmonar com 16 casos (Tabela 4).

A maior parte $(72 \%)$ dos casos de CCC neste estudo (82 casos) foram diagnosticados antes do nascimento. Para os nados-vivos, 54\% teve DPN, uma diferença estatisticamente significativa em comparação com as cardiopatias não críticas (27\%). As cardiopatias mais frequentemente diagnosticadas pré-natalmente (incluindo nados-vivos e IMG) foram as críticas: síndrome do coração esquerdo hipoplásico (12/12), d-transposição das grandes artérias (8/11), atrésia da artéria pulmonar (11/16), coartação da aorta (19/32 ou 59\%). Em 9\% das CCC o diagnóstico foi feito 'ao nascer', $15 \%$ na primeira semana de vida e $4 \%$ no restante período neonatal (três casos de coartação da aorta e um de atrésia da artéria pulmonar).

\section{Comparativo dos casos de interrupção médica da gra- videz versus nados vivos}

Todas as IMG neste estudo ( $n=84$ ) ocorreram com DPN de cardiopatia: $49 \%$ apresentava cardiopatia congénita crítica (em comparação com $20 \%$ no grupo sem IMG); no total $37 \%$ das cardiopatias congénitas críticas foram alvo de IMG, face a $13 \%$ das não críticas. Em $75 \%$ dos fetos sujeitos a interrupção da gravidez estavam presentes 
outras malformações associadas, enquanto nos casos sem interrupção apenas 1/4 tinha outras malformações (Tabela 4). Finalmente, $40 \%(n=34)$ apresentava anomalia cromossómica, em comparação com apenas $6 \%$ no grupo sem interrupção (Tabela 4). Todas estas diferenças tiveram significado estatístico (Tabela 5).

A maior parte dos casos de síndrome do coração esquerdo hipoplásico neste estudo foram alvo de interrupção da gravidez (8/11), bem como todos os casos de ventrículo direito de dupla saída (5/5). O maior número de IMG foi registado na região Norte, com diferença estatisticamente significativa (Tabela 5).

\section{Óbitos pós-natais}

Neste estudo registaram-se 15 óbitos num total de 339 nados-vivos: oito com DPN, seis com diagnóstico 'ao nascer', e um caso diagnosticado na primeira semana de transposição das grandes artérias (Tabela 6). Dos seis óbitos ocorridos na primeira semana de vida, quatro não tinham DPN; os casos 1, 2 e 3 ocorreram nas Regiões Autónomas.

\section{DISCUSSÃO}

A prevalência nacional de cardiopatias congénitas de 5:1000 nascimentos encontrada neste estudo por tipo de cardiopatia foi inferior aos dados publicados, ${ }^{1}$ com estimativas de 9,4:1000 ou valores superiores ${ }^{18-20}$; foi ainda inferior a dados previamente reportados no RENAC e na ilha de S. Miguel nos Açores. ${ }^{3,20}$ A comunicação interventricular (CIV) foi o defeito mais comum (38\% dos casos, correspondendo a $42 \%$ dos nados-vivos), seguida da comunicação

Tabela 5 - Resultados globais de comparação de IMG versus nados-vivos

\begin{tabular}{|c|c|c|c|c|}
\hline & \multirow{2}{*}{$\begin{array}{l}\text { Total } \\
423\end{array}$} & Interrupção gravidez & Nados-vivos (\%) & \multirow[b]{2}{*}{$p$} \\
\hline & & $84(20 \%)$ & $339(80 \%)$ & \\
\hline $\begin{array}{l}\text { 1. Norte } \\
\text { 2. Centro } \\
\text { 3. Lisboa/Vale Tejo } \\
\text { 4. Alentejo } \\
\text { 5. Algarve } \\
\text { 6. Açores } \\
\text { 7. Madeira }\end{array}$ & $\begin{array}{r}149(35 \%) \\
113(27 \%) \\
98(23 \%) \\
11(3 \%) \\
35(8 \%) \\
8(2 \%) \\
9(2 \%)\end{array}$ & $\begin{array}{r}43(29 \%) \\
17(15 \%) \\
15(15 \%) \\
0(0 \%) \\
9(25 \%) \\
0(0 \%) \\
0(0 \%)\end{array}$ & $\begin{array}{r}106(71 \%) \\
96(85 \%) \\
83(85 \%) \\
11(100 \%) \\
26(75 \%) \\
8(100 \%) \\
9(100 \%)\end{array}$ & \\
\hline $\begin{array}{l}\text { Sexo: } \\
\text { - Masculino } \\
\text { - Feminino }\end{array}$ & $\begin{array}{l}221(52 \%) \\
201(48 \%)\end{array}$ & $\begin{array}{l}41(49 \%) \\
42(51 \%)\end{array}$ & $\begin{array}{l}180(53 \%) \\
159(47 \%)\end{array}$ & $=0,62$ \\
\hline $\begin{array}{l}\text { Vigilância obstétrica (\%): } \\
\text { - Adequada } \\
\text { - DPN }\end{array}$ & $\begin{array}{l}339(81 \%) \\
195(46 \%)\end{array}$ & $\begin{array}{r}78(96 \%) \\
84(100 \%)\end{array}$ & $\begin{array}{l}279(78 \%) \\
111(33 \%)\end{array}$ & $\begin{array}{l}=0,001 \\
<0,001\end{array}$ \\
\hline Outras malformações & $148(35 \%)$ & $63(75 \%)$ & $85(25 \%)$ & $<0,001$ \\
\hline Cromossomopatias & $53(13 \%)$ & $34(40 \%)$ & $19(6 \%)$ & $<0,001$ \\
\hline Cardiopatias críticas & $109(26 \%)$ & $41(49 \%)$ & $68(20 \%)$ & $<0,001$ \\
\hline Tipo de cardiopatia: & & & & \\
\hline $\begin{array}{l}\text { 1. Shunt esquerdo-direito: } \\
\text { i. CIA/FOP } \\
\text { ii. CIV } \\
\text { iii. DSAV } \\
\text { iv. PCA } \\
\text { v. Truncus arteriosus }\end{array}$ & $\begin{array}{l}251(59 \%) \\
56(14 \%) \\
161(38 \%) \\
17(4 \%) \\
11(3 \%) \\
5(1 \%)\end{array}$ & $\begin{array}{r}4(5 \%) \\
18(21 \%) \\
4(5 \%) \\
0(0 \%) \\
1(1 \%)\end{array}$ & $\begin{array}{r}52(15 \%) \\
43(42 \%) \\
13(4 \%) \\
11(3 \%) \\
4(1 \%)\end{array}$ & \\
\hline $\begin{array}{l}\text { 2. Obstáculo esquerdo: } \\
\text { i. Coartação aorta } \\
\text { ii. Estenose aórtica }\end{array}$ & $\begin{array}{l}30(7 \%) \\
10(2 \%)\end{array}$ & $\begin{array}{r}8(10 \%) \\
1(1 \%)\end{array}$ & $\begin{array}{r}22(7 \%) \\
9(3 \%)\end{array}$ & \\
\hline $\begin{array}{l}\text { 3. Obstáculo direito } \\
\text { i. Estenose pulmonar }\end{array}$ & $22(5 \%)$ & $\begin{array}{l}3(4 \%) \\
\end{array}$ & $19(6 \%)$ & $<0,001$ \\
\hline 4. Transposição & $11(3 \%)$ & $6(7 \%)$ & $5(2 \%)$ & \\
\hline $\begin{array}{l}\text { 5. Complexa: } \\
\text { i. SCEH } \\
\text { ii. TOF } \\
\text { iii. Atrésia da artéria pulmonar } \\
\text { iv. RVPAT } \\
\text { v. VDDS }\end{array}$ & $\begin{array}{r}11(3 \%) \\
28(7 \%) \\
14(3 \%) \\
4(1 \%) \\
5(1 \%)\end{array}$ & $\begin{array}{r}8(10 \%) \\
7(8 \%) \\
5(6 \%) \\
0(0 \%) \\
5(6 \%)\end{array}$ & $\begin{array}{r}3(1 \%) \\
21(6 \%) \\
9(3 \%) \\
4(1 \%) \\
0(0 \%)\end{array}$ & \\
\hline 6. Outras & $29(8 \%)$ & $12(14 \%)$ & $17(5 \%)$ & \\
\hline
\end{tabular}

CIA: comunicação interauricular; CIV: comunicação interventricular; DSAV: defeito do septo auriculoventricular; PCA: persistência do canal arterial; SCEH: síndrome do coração esquerdo hipoplásico; TOF: tetralogia de Fallot; RVPAT: retorno venoso pulmonar anómalo total; VDDS: ventrículo direito de dupla saída 
Tabela 6 - Caracterização dos casos em que está registado óbito quanto a idade no óbito, diagnóstico prénatal (DPN), idade gestacional, peso ao nascer, diagnósticos cardíacos, presença de anomalias genéticas e outras malformações associadas.

\begin{tabular}{|c|c|c|c|c|c|c|c|}
\hline Caso & $\begin{array}{l}\text { Idade } \\
\text { óbito }\end{array}$ & DPN & $\begin{array}{c}\text { Idade } \\
\text { gestacional } \\
\text { (semanas) }\end{array}$ & $\begin{array}{c}\text { Peso ao } \\
\text { nascer } \\
(\mathrm{g})\end{array}$ & $\begin{array}{c}\text { Diagnóstico } \\
\text { cardíaco }\end{array}$ & $\begin{array}{l}\text { Anomalias } \\
\text { genéticas }\end{array}$ & $\begin{array}{c}\text { Outras } \\
\text { malformações }\end{array}$ \\
\hline 1 & $<8$ dias & Não* & 38 & 2300 & Estenose pulmonar & Não & Não \\
\hline 2 & $<8$ dias & Não* & 38 & 2760 & Estenose pulmonar & Não & Não \\
\hline 3 & $<8$ dias & Não* & 39 & 3200 & Estenose aórtica & Não & Não \\
\hline 4 & $<8$ dias & Não & 26 & 570 & $\mathrm{CIV}^{* *}$ & Não & Não \\
\hline 5 & 2 dias & Sim & 42 & 2155 & Tetralogia Fallot, PCA & Trissomia 18 & Sim \\
\hline 6 & 5 dias & Sim & 26 & 570 & $\mathrm{CIV}^{* *}$ & Não & Não \\
\hline 7 & 8 dias & Sim & 37 & 2643 & d-Transposição grandes artérias & Não & Não \\
\hline 8 & 9 dias & Sim & 37 & 2620 & DSAV, anomalia coronária, outras & Não & Não \\
\hline 9 & 10 dias & Sim & 38 & 3095 & Hipoplasia arco aórtico, CIV & Síndrome Di George & Não \\
\hline 10 & 22 dias & Sim & 38 & 2795 & Atrésia da artéria pulmonar & Síndrome Di George & Não \\
\hline 11 & 1 mês & Sim & 39 & 3035 & RVPAT, CIV & Não & Sim \\
\hline 12 & 1 mês & Não* & 37 & 1940 & RVPAT & Trissomia 18 & Sim \\
\hline 13 & 1 mês & Não & 38 & 2950 & d-Transposição grandes artérias & Não & Não \\
\hline 14 & 4 meses & Não* & 38 & 2800 & DSAV & Trissomia 21 & Não \\
\hline 15 & 9 meses & Sim & 32 & 1855 & Não especificado & Não & Não \\
\hline
\end{tabular}

*: Casos com diagnóstico 'ao nascer'; **: Fruto de gravidez gemelar

CIV: comunicação interventricular; PCA: persistência de canal arterial; DSAV: defeito do septo auriculoventricular; RVPAT: retorno venoso pulmonar anómalo total

interauricular (CIA) com $14 \%$ dos casos (correspondendo a $15 \%$ dos nados-vivos), tal como está descrito em todos os grandes estudos. ${ }^{2,18-20}$ No entanto, a persistência do canal arterial (PCA), habitualmente descrita como o terceiro diagnóstico mais frequente, neste caso foi o sétimo com apenas $3 \%$ do total de casos (ao invés dos habitualmente descritos $10 \%$ ). ${ }^{2,18,20,21} \mathrm{~A}$ coartação da aorta, tetralogia de Fallot e estenose da válvula pulmonar foram os diagnósticos seguintes mais frequentes, com a coartação da aorta mais frequente do que habitualmente descrito. ${ }^{1,2,18-20}$

A menor prevalência de cardiopatias congénitas poder-se-á dever ao carácter voluntário deste registo e a alguma assimetria entre instituições na notificação de casos. Alguns casos de cardiopatia com shunt-esquerdo direito (CIV, CIA, PCA) poderão ter sido detectados após o período neonatal, devido à diminuição das resistências vasculares pulmonares no final do primeiro mês de vida. A prevalência de cardiopatias críticas encontrada neste estudo também foi sobreponível aos valores publicados de cerca de $20 \%$ das cardiopatias, tal como a ausência de diagnóstico antes da alta hospitalar em cerca de $20 \%$ das CCC. ${ }^{22}$

As assimetrias regionais detectadas neste estudo podem ser explicadas, não só por diferentes graus de adesão ao registo voluntário, como pela assimetria de recursos altamente diferenciados como a ecocardiografia fetal e a orientação dos casos com DPN para serviços de Cardiologia Pediátrica (nas ARS do Norte, LVT ou Coimbra). ${ }^{23}$ As regiões com menor densidade populacional como o Alentejo, ou mais isoladas, como as regiões autónomas, além de terem tido um menor número de nascimentos, poderão ter menor acesso a estes recursos, e como tal menor número de DPN de cardiopatias.
A mortalidade observada de 17,5 por 100000 nascimentos neste estudo é similar à de dados internacionais publicados..$^{11}$ No entanto este valor poderá estar subvalorizado, tendo em conta que três dos quatro centros cirúrgicos nacionais não efetuaram qualquer notificação. A extrema prematuridade, presença de anomalias genéticas, outras malformações associadas e a localização geográfica mais periférica (casos 1, 2 e 3 na Tabela 6) associaram-se a um pior prognóstico.

A codificação dos diagnósticos foi feita com base na ICD-10 e revista pelos investigadores do INSA, conferindo homogeneidade. Os resultados encontrados neste estudo são um importante contributo para o conhecimento da realidade nacional e poderão contribuir para o planeamento e estratégias de organização do diagnóstico pré-natal e rastreio de cardiopatias congénitas críticas em recém-nascidos.

Relativamente ao rastreio neonatal de cardiopatias congénitas por oximetria de pulso, estima-se que até $20 \%$ dos doentes com CCC terão alta da maternidade sem diagnóstico da sua cardiopatia, pré ou pós-natal. ${ }^{22}$ Para colmatar estas altas sem diagnóstico, na última década tem sido estudada e implementada a oximetria de pulso como método de rastreio universal de CCC nos recém-nascidos antes da alta da maternidade (idealmente após as 24 horas de vida). ${ }^{5,24-26}$ Algumas das vantagens deste método são a sua rapidez e facilidade de realização "à cabeceira do doente", além da elevada sensibilidade na detecção das CCC cianóticas, ${ }^{4}$ consideradas o alvo primário do rastreio (Tabela 1). No entanto, o rastreio de CCC por oximetria de pulso apresenta limitações importantes, nomeadamente falsos positivos e menor sensibilidade para cardiopatias 
acianóticas como a coartação da aorta. .,27 $^{-27}$

A título de exemplo, nos Estados Unidos da América (EUA), e países nórdicos, este método de rastreio tem sido implementado, e tem sido reportada diminuição da mortalidade e do referido gap diagnóstico. ${ }^{11}$ No entanto, no Reino Unido foi realizado um estudo prospectivo multicêntrico (32 836 rastreios) que identificou 239 recém-nascidos com hipoxémia, oito com CCC, e dois falsos negativos; não foi tomada a decisão de implementar o rastreio universal, tendo em conta que a maior parte dos rastreios positivos não tinham doença cardíaca, desviando recursos de recém-nascidos em necessidade de maiores cuidados. ${ }^{28}$

Várias maternidades portuguesas, públicas e privadas, têm implementado este rastreio aos recém-nascidos antes da alta. ${ }^{29}$ No entanto a realidade nacional é bastante diferente dos países acima referidos: os recém-nascidos geralmente têm alta no segundo dia de vida (ou depois), e são sempre observados por um médico Pediatra, ao contrário dos EUA e países nórdicos em que são avaliados por enfermeiros. Do ponto de vista dos autores, este tipo de rastreio poderá ser implementado a nível populacional, apenas depois de um levantamento mais extenso da realidade nacional, nomeadamente dos potenciais ganhos em saúde que seriam obtidos.

O carácter voluntário dos registos no RENAC é a principal limitação deste estudo, com uma provável subvalorização dos casos. Poder-se-ão também verificar assimetrias na forma como são registados os diagnósticos nas diferentes instituições. A análise dos dados de vários anos consecutivos ou de diferentes coortes poderá ajudar a compreender as tendências na evolução dos indicadores agora descritos. Em próximos estudos será igualmente importante que a recodificação dos diagnósticos na base de dados seja realizada por mais do que um médico. Realçamos que este é, tanto quanto resultou da revisão da literatura, o primeiro trabalho publicado no âmbito nacional com estimativas da prevalência das diferentes cardiopatias congénitas e incluindo contributos de todas as maternidades.

\section{REFERÊNCIAS}

1. Hoffman JIE, Kaplan S. The incidence of congenital heart disease. J Am Coll Cardiol. 2002;39:1890-900.

2. Van Der Linde $D$, Konings EE, Slager MA, Witsenburg M, Helbing WA, Takkenberg JJ, et al. Birth prevalence of congenital heart disease worldwide: a systematic review and meta-analysis. J Am Coll Cardiol. 2011;58:2241-7.

3. Braz P, Machado A, Dias CM. Registo Nacional de Anomalias Congénitas: relatório 2014/2015. Instituto Nacional de Saúde Doutor Ricardo Jorge, editor. Lisboa: Ministério da Saúde; 2017.

4. Mahle WT, Newburger JW, Matherne GP, Smith FC, Hoke TR, Koppel R, et al. Role of pulse oximetry in examining newborns for congenital heart disease: a scientific statement from the American Heart Association and American Academy of Pediatrics. Circulation. 2009;120:447-58.

5. Thangaratinam S, Brown K, Zamora J, Khan KS, Ewer AK. Pulse oximetry screening for critical congenital heart defects in asymptomatic newborn babies: a systematic review and meta-analysis. Lancet. 2012;379:2459-64.

6. Plana M, Zamora J, Suresh G, Fernandez-Pineda L, Thangaratinam S, Ewer A. Pulse oximetry screening for critical congenital heart defects. Cochrane Database Syst Rev. 2018;3:CD011912.

7. Direcção Geral da Saúde. Programa Nacional para a Vigilância da

\section{CONCLUSÃO}

Em 2015 foi registada no RENAC uma prevalência de cardiopatias congénitas em Portugal de 5:1000 nascimentos, com assimetrias regionais importantes. Em 20\% dos nados-vivos o defeito cardíaco era crítico. Dos 15 óbitos observados neste estudo, apenas um teve diagnóstico tardio da sua cardiopatia congénita (após a alta hospitalar). Os dados deste estudo são relevantes para o conhecimento da realidade nacional e para um melhor planeamento dos Cuidados de Saúde. É importante uma maior adesão ao Registo Nacional de Anomalias Congénitas (RENAC), e monitorização dos resultados, em articulação com as sociedades médicas das especialidades envolvidas, visando o mais completo conhecimento da realidade nacional na área das cardiopatias congénitas, um adequado planeamento dos cuidados, e eventual implementação de rastreio universal e monitorização dos resultados.

\section{PROTECÇÃO DE PESSOAS E ANIMAIS}

Os autores declaram que os procedimentos seguidos estavam de acordo com os regulamentos estabelecidos pelos responsáveis da Comissão de Investigação Clínica e Ética e de acordo com a Declaração de Helsínquia da Associação Médica Mundial.

\section{CONFIDENCIALIDADE DOS DADOS}

Os autores declaram ter seguido os protocolos do seu centro de trabalho acerca da publicação de dados.

\section{CONFLITOS DE INTERESSE}

Os autores declaram não ter conflitos de interesse relacionados com o presente trabalho.

\section{FONTES DE FINANCIAMENTO}

Este trabalho não recebeu qualquer tipo de suporte financeiro de nenhuma entidade no domínio público ou privado.

Gravidez de Baixo Risco. Lisboa: DGS; 2015

8. Schultz AH, Localio AR, Clark BJ, Ravishankar C, Videon N, Kimmel SE. Epidemiologic features of the presentation of critical congenital heart disease: implications for screening. Pediatrics. 2008;121:751-7.

9. Ailes EC, Gilboa SM, Honein MA, Oster ME. Estimated number of infants detected and missed by critical congenital heart defect screening. Pediatrics. 2015;135:1000-8.

10. Mahle WT, Martin GR, Beekman RH, Morrow WR, Rosenthal GL, Snyder $\mathrm{CS}$, et al. Endorsement of health and human services recommendation for pulse oximetry screening for critical congenital heart disease. Pediatrics. 2012;129:190-2.

11. Abouk R, Grosse SD, Ailes EC, Oster ME. Association of US State implementation of newborn screening policies for critical congenital heart disease with early infant cardiac deaths. Jama. 2017;318:2111.

12. Khairy P, lonescu-Ittu R, MacKie AS, Abrahamowicz M, Pilote L, Marelli AJ. Changing mortality in congenital heart disease. J Am Coll Cardiol. 2010;56:1149-57.

13. Marelli AJ, Ionescu-Ittu R, Mackie AS, Guo L, Dendukuri N, Kaouache M. Lifetime prevalence of congenital heart disease in the general population from 2000 to 2010. Circulation. 2014;130:749-56.

14. Braz P, Machado A, Dias CM. Registo Nacional de Anomalias 
Congénitas. Relatório 2011-2013. Lisboa: Instituto Nacional de Saúde Doutor Ricardo Jorge; 2015.

15. Comissão de Ética para a Investigação Clínica. Documento CEIC sobre o Regulamento Geral de Proteção de Dados (RGPD) no contexto da Investigação Clínica. 2018. [acedido 2019 out 8]. Disponível em: https:// www.ceic.pt/documents/20727/57550/RGPD/.

16. Instituto Nacional de Estatística. Portal do INE - Nados-vivos 2015. 2015 [acedido 2019 out 8]. Disponível em: https://www.ine.pt/xportal/ xmain?xpid=INE\&xpgid=ine_indicadores\&indOcorrCod=0008234\&cont exto=pi\&selTab=tab0.

17. Instituto Nacional de Estatística. Estatísticas vitais - Óbitos fetais 2015. [acedido 2019 out 8]. Disponível em: https://www.ine.pt/xportal/ xmain?xpid=INE\&xpgid=ine_indicadores\&indOcorrCod=0008173\&cont exto=bd\&selTab=tab2.

18. Liu Y, Chen S, Zu L, Black GC, Choy M, Li N, et al. Early childhood global birth prevalence of congenital heart defects 1970 - 2017: updated systematic review and meta-analysis of 260 studies. Int J Epidemiol. 2019;48:455-63.

19. Leirgul E, Fomina T, Brodwall K, Greve G, Holmstrøm H, Vollset SE, et al. Birth prevalence of congenital heart defects in Norway 1994-2009 - a nationwide study. Am Heart J. 2014;168:956-64.

20. Cymbron T, Anjos R, Cabral R, Macedo C, Duarte CP, Mota-Vieira L. Epidemiological characterization of congenital heart disease in São Miguel Island, Azores, Portugal. Community Genet. 2006;9:107-12.

21. Hoffman JI, Kaplan S. The incidence of congenital heart disease. J Am Coll Cardiol. 2002;39:1890-900.

22. Riede FT, Wörner C, Dähnert I, Möckel A, Kostelka M, Schneider P.
Effectiveness of neonatal pulse oximetry screening for detection of critical congenital heart disease in daily clinical routine-results from a prospective multicenter study. Eur J Pediatr. 2010;169:975-81.

23. Divisão de Saúde Materno-Infantil e dos Adolescentes. Saúde MaternoInfantil: Rede de Referenciação Materno-Infantil. Lisboa: DirecçãoGeral da Saúde; 2001.

24. Kemper AR, Mahle WT, Martin GR, Cooley C, Kumar P, Morrow R, et al. Strategies for implementing screening for critical congenital heart disease. Pediatrics. 2011;128:1259-67.

25. Sánchez Luna M, Pérez Muñuzuri A, Sanz López E, Leante Castellanos $\mathrm{JL}$, Benavente Fernández I, Ruiz Campillo CW, et al. Cribado de cardiopatías congénitas críticas en el periodo neonatal. Recomendación de la Sociedad Española de Neonatología. An Pediatría. 2018;88:112. e1-6.

26. Manzoni P, Martin GR, Sanchez Luna M, Mestrovic J, Simeoni U, Zimmermann $\mathrm{L}$, et al. Pulse oximetry screening for critical congenital heart defects: a European consensus statement. Lancet Child Adolesc Heal. 2017;1:88-90.

27. Granelli AD, Wennergren M, Sandberg K, Mellander M, Bejlum C, Inganäs $L$, et al. Impact of pulse oximetry screening on the detection of duct dependent congenital heart disease: a Swedish prospective screening study in 39821 newborns. BMJ. 2009;338:145-8.

28. Evans C. Newborn pulse oximetry screening pilot update. 2017 [acedido 2020 mai 08]. Disponível em: https://phescreening.blog.gov. uk/2017/01/10/newborn-pulse-oximetry-screening-pilot-update/.

29. Morais S, Mimoso G. Oximetria de pulso no diagnóstico de cardiopatia congénita. Acta Pediátrica Port. 2014;44:343-7. 